\title{
Model completion and validation using inversion of grey box models
}

\author{
Bill Whiten ${ }^{1}$
}

(Received 1 October 2012; revised 26 March 2013)

\begin{abstract}
A grey box model uses some theoretical structure that is not complete and it is thus necessary to complete the model using data. Unfortunately, as the models are generally nonlinear, the efficient linear methods of selecting regression terms were not available for the completion of these models. However, the method of model inversion converts the unknown parts within a nonlinear model into an approximate linear model such that efficient linear term selection methods can be applied. In addition to completing grey box models, the inversion technique can be used to test if the model adequately describes the available data. Subject class: 62-07, 62J02, 62J05

Keywords: grey box models, model completion, model validation, model inversion

http://journal austms.org.au/ojs/index.php/ANZIAMJ/article/view/6125 gives this article, (c) Austral. Mathematical Soc. 2013. Published May 21, 2013, as part of the Proceedings of the 16th Biennial Computational Techniques and Applications Conference. ISSN 1446-8735. (Print two pages per sheet of paper.) Copies of this article must not be made otherwise available on the internet; instead link directly to this URL for
\end{abstract} this article. 


\section{Contents}

1 Introduction

C188

2 Examples of grey box models

C190

2.1 Ore grinding mills . . . . . . . . . . . . . . . C190

2.2 Hydrocyclone . . . . . . . . . . . . . . . . . . . . C190

3 Linear regression

C191

4 The grey box model

C193

4.1 Inversion of the grey box model . . . . . . . . . . . . C C193

4.2 Model inversion and linear regression . . . . . . . . . C194

5 Model validation

C195

6 Conclusions

C196

References

C197

\section{Introduction}

Grey box models are an important compromise between purely theoretical models and purely empirical models. An incomplete theoretical basis is completed by some additional experimental information. Such a model varies from a theoretical model where some parameter values are determined from data, to models with a very minimal theoretical structure with the majority of the remaining model coming from experimental data. As most models fall within this range of types the construction and validation of grey box models is important. Section 2 briefly describes two grey box models commonly used in simulation of mineral processing plants.

There is a large and useful theory on the construction of models using linear 
regression, which is far more efficient than the more limited range of techniques that are available for the construction of nonlinear models, which typically make extensive use of nonlinear least squares [9]. In the case where model parameters are calculated from individual data sets, typically by nonlinear least squares, it is then possible to use linear regression to find relations for the model parameters that can be included into the model. While this is often the final step, it is preferable to then fit the nonlinear model over all the data sets, and possibly further refine the model [12]. For the case where the parameters cannot be calculated from each data set it was necessary to test each possible form of the model separately. This approach, relying on nonlinear optimisation of individual cases, is used in the Matlab system identification toolbox (grey box model identification ${ }^{1}$ ), and by Bohlin [1], who included a description of the MoCaVa program for grey box modelling. Kojovic and Whiten $[5,4,14]$ noted that if the model residuals are random there is little prospect of improving the model. They used linear regression to determine if there was any non-random behaviour in the residuals. Kojovic [4] tested any terms that were useful in predicting residuals within the model structure to determine if they provided a useful improvement in model accuracy.

Some important properties of linear regression are given in Section 3, and Section 4 shows how a grey box model is inverted, so that the unknown internal parts of a nonlinear model can be analysed using linear techniques. The same inversion method is used to perform validation tests that determine if a model adequately describes the available data. In Section 5 we discuss model validation and how model inversion is used to determine if a model can be improved.

\footnotetext{
${ }^{1}$ http://www . mathworks.com/help/ident/grey-box-model-estimation.html
} 


\section{Examples of grey box models}

There are many possible examples of grey box models. Below are two example models from mineral processing. In each case there is a known structure to the model and the behaviour of certain parameters depends in an unknown manner on some operating conditions. The operating conditions are assumed to include all the variables that affect the system being modelled, possibly including some that are recorded but not easily changed.

\subsection{Ore grinding mills}

Ore grinding mills are used to reduce ore to fine particles for separation of the valuable component from the waste. The particles are described by a vector giving mass flow in size fractions. The breakage of the ore particles is described by a matrix that describes how, after breakage, the broken particle appear in finer size fractions. Two parameters, the rate of breakage and rate of discharge, combine into a ratio with values for each size fraction. The grinding mill is described by [13]

$$
\mathbf{p}=\left[I+(I-A) R D^{-1}\right]^{-1} \mathbf{f},
$$

where $\mathbf{f}$ is the vector of mass rate in size fraction of mill feed, $\mathbf{p}$ is the vector of mass rate in size fraction of mill product, $A$ is a known triangular matrix giving the appearance after breakage, and $R D^{-1}$ is the ratio of rates in a diagonal matrix. In this model the elements of $R^{-1}$ vary in an unknown manner with operating conditions that include feed rate, ore hardness, power input and water input.

\subsection{Hydrocyclone}

Hydrocyclones are used to separate fine particles from coarse particles. For this case the theory is limited to the observation that similar sized particles 
will behave in a similar manner. A smooth graph giving the fraction of a particle size that reports to the fine product can be drawn. The curve is [8]

$$
p(s)=c \frac{e^{a s / d}-1}{e^{a s / d}+e^{a}-2} f(s),
$$

where $s$ is the particle size, $f(s)$ is the mass flow of feed of size $s, p(s)$ is the mass flow of fine product of size $s, a$ is a parameter for the sharpness of the curve, $\mathrm{c}<1$ is a parameter for height of the curve, and $\mathrm{d}$ is a parameter for width of the curve (size of $c / 2$ separation). The three parameters $a$, $c$ and $d$ vary in a complex manner with operating conditions that include hydrocyclone dimensions, feed rate, and water content.

\section{Linear regression}

Model inversion is the initial step in the proposed model completion. Model inversion converts the internal nonlinear components into a linear form that is analysed by linear regression. Some important properties of linear regression relevant to the model inversion are reviewed in this section.

Linear regression uses

$$
y=\sum_{j} x_{j} a_{j}
$$

to predict $y$ from values of $x_{j}$, using the linear coefficients $a_{j}$ determined from data. Linear regression efficiently determines which of the $a_{j}$ values are nonzero and determines their values $[2,11,7]$. The $\boldsymbol{x}_{\boldsymbol{j}}$ can be simple measured values, or can be functions of the simple values allowing the generation of nonlinear responses.

Given a vector of data values for $\mathbf{y}$ and a matrix $\mathbf{X}$ with rows containing data corresponding to the element of $\mathbf{y}$, regression determines a by minimising the sum of squares

$$
(\mathbf{y}-X \mathbf{a})^{\top}(\mathbf{y}-X \mathbf{a}) \text {. }
$$


To do this, first factor $\mathbf{X y}$ as

$$
\mathrm{Q}\left[\begin{array}{cc}
\mathrm{U} & \mathrm{u} \\
0 & v
\end{array}\right]
$$

where $\mathrm{Q}$ is orthogonal and $\mathrm{U}$ is upper triangular [7]. Then solve

$$
\mathrm{u}^{\top} \mathbf{a}=\mathbf{u}
$$

for $\mathbf{a}$, which is easily done as $U^{\top}$ is lower triangular. This is a more efficient approach to using matrix inversion in the common formula $\mathbf{a}=\left(X^{\top} \mathbf{X}\right)^{-1} X^{\top} \mathbf{y}$. Here, $U^{\top} U=X^{\top} X$ and $U$ are also obtained using the Cholesky factorisation of $X^{\top} X$.

The sum of squares (2) is equal to $v^{2}$ in equation (3), thus it is not necessary to go as far as calculating a to determine the accuracy of the regression. If an extra variable is added as a column to the right side of $X$ it is only necessary to add an extra column to $\boldsymbol{U}$ and update the values of $u$ and $v$ to get the new sum of squares. Various criteria (e.g., AIC, BIC, CIC [3, 6]) are available to determine if the sum of squares is reduced sufficiently for the variable and its corresponding coefficient make a useful contribution to the model accuracy. The variable is omitted if it does not make a useful contribution.

Another method for selecting variables that make a useful contribution to the reduction of the sum of squares is to bias the coefficients towards zero, so that variables with little effect on the sum of squares become close to zero, and can be removed. The simplest way to do this is to add $a_{j}^{2}$ to the sum of squares, so that the regression requires minimising the sum of squares of

$$
\left[\begin{array}{c}
X \\
\sigma \mathrm{I}
\end{array}\right] \mathbf{a}-\left[\begin{array}{l}
\mathbf{y} \\
\mathbf{0}
\end{array}\right]
$$

If there are many possible columns (potential variables) and few rows (data sets), the following is used to reduce the computation:

$$
\left(\sigma^{2} I+X^{\top} X\right)^{-1}=\frac{1}{\sigma^{2}}\left[I-X^{\top}\left(\sigma^{2} I+X X^{\top}\right)^{-1} X\right]
$$


For many more columns than rows the matrix inversion on the right hand side of equation (6) (and also its factorisation) is much less work than the matrix inversion on the left.

\section{The grey box model}

A general form of the grey box model is

$$
\mathbf{m}(\mathbf{f}, \mathbf{p}, \mathbf{q})=\mathbf{0} \quad \text { with } \quad \mathbf{q}=A \mathbf{c}
$$

where $\mathbf{f}$ is a vector giving inputs or feed to model, $\mathbf{p}$ is a vector giving outputs or product from model, the vector function $\mathbf{m}$ is the known model which provides a set of conditions, $\mathbf{q}$ is the vector of parameters in the model, $\mathbf{c}$ is the vector of operating conditions, and $A$ is a matrix of unknown coefficients.

The model is manipulated into a form where the elements of the vector $\mathbf{m}$ are suitable for least square fitting. For example, by writing the model as the weighted difference between the model output vector and the predicted output vector. As with the linear models discussed above, the values in $\mathbf{c}$ can be transforms of the basic operating conditions so that $\mathbf{q}$ is not restricted to linear behaviour.

\subsection{Inversion of the grey box model}

To turn a particular grey box model structure into a usable model some sets of data $f_{k}^{*}, p_{k}^{*}, c_{k}^{*}$ are needed. Using these data sets, it is necessary to determine the zero and nonzero elements of $A$.

Because of some complexity in the notation, the model inversion technique is given for the simple case of one data set. Using an initial estimate $\mathbf{q}^{*}$ for $\mathbf{q}$ the 
model residual is expanded using the first terms of a series in $\delta \mathbf{q}=\mathbf{q}-\mathbf{q}^{*}$,

$$
\begin{aligned}
\mathbf{m}\left(\mathbf{f}^{*}, \mathbf{p}^{*}, \mathbf{q}\right) & =\mathbf{m}\left(\mathbf{f}^{*}, \mathbf{p}^{*}, \mathbf{q}^{*}+\delta \mathbf{q}\right) \\
& \approx \mathbf{m}\left(\mathbf{f}^{*}, \mathbf{p}^{*}, \mathbf{q}^{*}\right)+\mathbf{m}^{\prime}\left(\mathbf{f}^{*}, \mathbf{p}^{*}, \mathbf{q}^{*}\right) \delta \mathbf{q}
\end{aligned}
$$

where $\mathbf{m}^{\prime}$ is the derivative of $\mathbf{m}$ with respect to $\mathbf{q}$, that is

$$
\mathbf{m}^{\prime}\left(\mathbf{f}^{*}, \mathbf{p}^{*}, \mathbf{q}^{*}\right)=\frac{\partial \mathbf{m}\left(\mathbf{f}^{*}, \mathbf{p}^{*}, \mathbf{q}^{*}\right)}{\partial \mathbf{q}^{* \top}} .
$$

Then, instead of using linear least squares to estimate $\delta \mathbf{q}$, as would normally be done, we eliminate $\delta \mathbf{q}$ using $\delta \mathbf{q}=\mathbf{q}-\mathbf{q}^{*}$ and then eliminate $\mathbf{q}$ using $\mathbf{q}=A \mathbf{c}^{*}$ to obtain

$$
\begin{aligned}
\mathbf{m}\left(\mathbf{f}^{*}, \mathbf{p}^{*}, \mathbf{q}\right) & \approx \mathbf{m}\left(\mathbf{f}^{*}, \mathbf{p}^{*}, \mathbf{q}^{*}\right)+\mathbf{m}^{\prime}\left(\mathbf{f}^{*}, \mathbf{p}^{*}, \mathbf{q}^{*}\right)\left(A \mathbf{c}-\mathbf{q}^{*}\right) \\
& =\mathbf{m}\left(\mathbf{f}^{*}, \mathbf{p}^{*}, \mathbf{q}^{*}\right)-\mathbf{m}^{\prime}\left(\mathbf{f}^{*}, \mathbf{p}^{*}, \mathbf{q}^{*}\right) \mathbf{q}^{*}+\mathbf{m}^{\prime}\left(\mathbf{f}^{*}, \mathbf{p}^{*}, \mathbf{q}^{*}\right) A \mathbf{c}^{*}
\end{aligned}
$$

Here, the first two terms are a known constant vector, while the last term is linear in the elements of $A$ with known constant coefficients. Hence, equation (10) is in the form where linear regression can be used to determine the elements of $A$.

\subsection{Model inversion and linear regression}

Equation (10) has the necessary linear form for linear regression, but is not yet expressed in the vector form commonly used in linear regression. Comparing the regression form $\mathbf{X} \mathbf{a} \approx \mathbf{b}$ with equation (10), expanded for multiple data sets $f_{k}^{*}, p_{k}^{*}, c_{k}^{*}$ and the estimates $q_{k}^{*}$, gives

$$
X=\left[\begin{array}{ccc}
c_{1,1} M_{1} & c_{2,1} M_{1} & \cdots \\
c_{1,2} M_{2} & c_{2,2} M_{2} & \cdots \\
\vdots & \vdots & \ddots
\end{array}\right], \quad \mathbf{a}=\left[\begin{array}{c}
a_{1} \\
a_{2} \\
\vdots
\end{array}\right], \quad \mathbf{y}=\left[\begin{array}{c}
\mathbf{m}\left(f_{1}^{*}, p_{1}^{*}, q_{1}^{*}\right)-M_{1} q_{1}^{*} \\
\mathbf{m}\left(f_{2}^{*}, p_{1}^{*}, q_{2}^{*}\right)-M_{2} q_{2}^{*} \\
\vdots
\end{array}\right]
$$


where

$$
M_{k}=\mathbf{m}^{\prime}\left(f_{k}^{*}, p_{k}^{*}, q_{k}^{*}\right)=\left[\frac{\partial m_{i}\left(f_{k}^{*}, p_{k}^{*}, q_{k}^{*}\right)}{\partial q_{j}^{*}}\right],
$$

$A=\left[a_{1} a_{2} \cdots\right]$ and $\left[c_{i, j}\right]=\left[c_{1}^{*} c_{2}^{*} \cdots\right]$. Once these substitutions are made, standard linear regression analysis techniques are applied.

\section{Model validation}

Model validation, by testing whether the model can be improved using experimental data, is considered in this section, although testing the model against experimental data is not the only approach. For instance, a model can be examined for behaviour consistent with the expected responses. In addition, it may be more relevant to ask if the model is sufficient for its proposed application. The required accuracy assists in determining whether an increase in model complexity is justified by the associated increase in accuracy.

Tests such as chi-squared on the residuals are not of much use, as they depend critically on the estimates of the standard deviations of the residuals, which are seldom known with sufficient accuracy. Further, if the chi-squared test fails, as it often does, then it does not provide any useful information on what to do next.

A more useful approach is to determine if the residuals can be predicted, possibly after sorting into the different types of residuals present (e.g. error in water flow and error in ore flow). If the operating conditions do not predict the residuals, then the residuals may well be essentially random, and it is unlikely that the model can be improved. However, if the residuals can be predicted, then the operating conditions useful for predicting the residues are those that need to be used to improve the model.

The relevance of the residual prediction needs to be considered. A small but 
consistent bias in the residuals may be highly statistically significant but in practice irrelevant. Similarly, when there is a lot of data very significant statistical predictions can indicate improvements in the potential accuracy of the model that are in practice so small as to be quite irrelevant.

The model inversion technique allows an examination of the predicability of the model residuals to be combined with the direct improvement of the model. It can be used as a test for an existing model to see if useful improvements can be made, or as an independent test on a separate set of data. Extra operating conditions or transforms of existing operating conditions can be introduced to investigate possible extensions of the model.

When developing or testing using regression techniques it is necessary to be aware of the possibility of over fitting, particularly when term selection is from a large collection of possible operating conditions. When possible, it is highly desirable to withhold some data for an independent test of the model structure and accuracy.

\section{Conclusions}

The grey box model $\mathbf{m}(\mathbf{f}, \mathbf{p}, \mathbf{q})=\mathbf{0}$ with $\mathbf{q}=$ Ac is linearised with respect to parameter $\mathbf{q}$, using $\delta \mathbf{q}$, to allow the use of efficient linear regression techniques to determine the structure and values of $A$. This approach was demonstrated by Xiao [15, chapters 8 and 9] for both conventional and more complex types of hydrocyclone models. He showed, using cross validation, that the models developed were more accurate than those proposed elsewhere. The model inversion technique is yet to be applied to ball mills as sufficient industrial data is not readily available, due to the difficulties in sampling large scale units sufficiently accurately, and issues with much of the existing data being proprietary.

Model inversion combined with regression techniques sometimes seems to be more robust than nonlinear least squares, as term selection ensures calculation 
is well conditioned. Model validation is approached by showing residuals are not able to be predicted and thus the model is not able to be improved. When the residuals can be predicted the model inversion provides a method which determines how the model should be extended and improved. The Matlab functions greyboxeval for model evaluation, and greyboxbuild for model completion are available from the Matlab file exchange. ${ }^{2}$

Acknowledgements This work was developed at the Julius Kruttschnitt Mineral Research Centre, The University of Queensland. Ray Phillips, Ricardo Pascual, and Jinhong Xiao worked on developing programs to implement model inversion. Jinhong Xiao [15] also showed how the techniques is applied to an advanced hydrocyclone model and Tim Tran [10] undertook related work on term selection in linear regression. The particular version of the Woodbury formula comes from a presentation given by Matt Wald.

\section{References}

[1] Bohlin, T. P., Practical grey-box process identification, Theory and applications (Advances in industrial control, Springer (London), 2006. ISBN: 978-1-846-28402-1. C189

[2] Draper, N. R., and Smith, H., Applied regression analysis, Wiley (New York), 1998. ISBN: 978-0-471-17082-2. C191

[3] Gustafsson, F., and Hjalmarsson, H., Twenty-one ML estimators for model selection, Automatica, 31(10) 1377-1392, 1995. doi:10.1016/0005-1098(95)00058-5 C192

[4] Kojovic, T., The davelopment and application of Model-an automated model builder for mineral processing, $\mathrm{PhD}$ thesis, The University of Queensland, 1989. C189 
[5] Kojovic, T., and Whiten W. J., Evaluation of the quality of simulation models, Innovations in mineral processing, (Lauretian University, Sudbury) p437-446, 1994. ISBN: 088667025X. C189

[6] Konishi, S., and Kitagawa, G., Information criteria and statistical Modeling, Springer (New York) 2010. ISBN 978-1-441-92456-8 C192

[7] Lawson, C. L., and Hanson, R. J., Solving least squares problems, SIAM (Philadelphia), 1995. ISBN: 978-0-898-71356-5. C191, C192

[8] Napier-Munn, T. J., Morrell, S., Morrison, R. D. and Kojovic, T., Mineral comminution circuits-their operation and optimisation, Julius Kruttschnitt Mineral Research Centre (Brisbane), 1996. C191

[9] Press, W. H., Teukolsky, S. A., Vetterling, W. T., and Flannery, B. P., Numerical recipes, Cambridge (New York), 2007. ISBN:

978-0-521-88068-8. C189

[10] Tran, V., Improved calibration techniques for nuclear analysers, $\mathrm{PhD}$ thesis, The University of Queensland, 1998. C197

[11] Weisburg, S., Applied linear regression, Wiley-Interscience (Hoboken, N.J.), 2005. ISBN: 978-0-471-66379-9. C191

[12] Whiten, W. J., Model building techniques applied to mineral treatment processes, Symp. on Automatic Control Systems in Mineral Processing Plants, (Australas. Inst. Min. Metall., S. Queensland Branch, Brisbane), 129-148, 1971. C189

[13] Whiten, W.J., A matrix theory of comminution machines, Chem. Eng. Sci., 29, 589-599., 1974. doi:10.1016/0009-2509(74)80070-9. C190

[14] Whiten, W. J., Determination of parameter relations within non-linear models, SIGNUM Newsletter, 29(3-4), 2-5, 1994.

doi:10.1145/192527.192535. C189 
[15] Xiao, J., Extensions of model building techniques and their applications in mineral processing, $\mathrm{PhD}$ thesis, The University of Queensland, 1998. C196, C197

\section{Author address}

1. Bill Whiten, 4 Magnet Cl, Riverhills, Brisbane, 4072, Australia. mailto:billwhiten@tpg.com.au 